\title{
$\begin{array}{r}\text { WAGENINGEN } \\ \hline \\ \hline\end{array}$
}

\author{
Online and in situ analysis of organs-on-a-chip \\ Santbergen, M. J. C., van der Zande, M., Bouwmeester, H., \& Nielen, M. W. \\ $\mathrm{F}$.
}

This is a "Post-Print" accepted manuscript, which has been published in "TrAC Trends in Analytical Chemistry"

This version is distributed under a non-commercial no derivatives Creative Commons

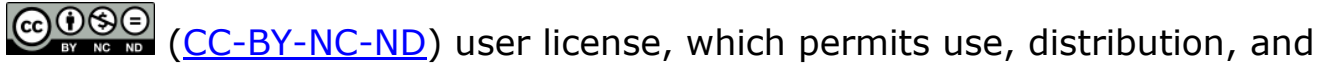
reproduction in any medium, provided the original work is properly cited and not used for commercial purposes. Further, the restriction applies that if you remix, transform, or build upon the material, you may not distribute the modified material.

Please cite this publication as follows:

Santbergen, M. J. C., van der Zande, M., Bouwmeester, H., \& Nielen, M. W. F. (2019). Online and in situ analysis of organs-on-a-chip. TrAC - Trends in Analytical Chemistry, 115, 138-146. https://doi.org/10.1016/j.trac.2019.04.006 


\section{Online and in situ analysis of Organs-on-a-chip}

Milou J.C. Santbergen ${ }^{\mathrm{a}, \mathrm{b}}$ Meike van der Zande ${ }^{\mathrm{c}}$, Hans Bouwmeester ${ }^{\mathrm{d}}$, Michel W.F. Nielen ${ }^{\mathrm{a}, \mathrm{c}}$

a Laboratory of Organic Chemistry, Wageningen University, Stippeneng 4, 6708 WE

Wageningen, The Netherlands

b TI-COAST, Science Park 904, 1098 XH Amsterdam, The Netherlands

${ }^{\mathrm{C}}$ RIKILT, Wageningen Research, P.O. Box 230, 6700 AE Wageningen, The Netherlands

${ }^{d}$ Division of Toxicology, Wageningen University, Stippeneng 4, 6708 WE Wageningen, The

Netherlands

\section{Highlights}

- Organs-on-a-chip are used for unravelling biological processes like, homeostasis, metabolism and responses to stimuli.

- Monitoring the microenvironment is crucial for establishing relevant biological organ-ona-chip models.

- Online and in situ analysis of organ-on-a-chip systems allows for automated and realtime analysis of biological processes.

- Biological integrity needs to be preserved when interfacing organ-on-a-chip models with sensors and high-end instruments.

\section{Abstract}

Organ-on-a-chip technology is used to study biological processes that involve multiple cell types and temporal changes like, homeostasis, metabolism of compounds and responses to chemical triggers. Main benefits of organ-on-a-chip systems include: improved mimicking of 
the in vivo situation, easy manipulation of the microenvironment and low reagent consumption. Exploiting the unique dynamic aspects of organ-on-a-chip technology, such as liquid flow, automated online measurement of parameters by sensors or online coupling to analytical equipment becomes feasible. Apart from the challenge to detect drug uptake and chemical changes in real-time with high resolution at the microscale, the biggest challenge, is the detection of the analyte of interest in cell culture medium, as this contains high amounts of salts, sugars and proteins required by the living cells. In this review online and in situ analytical techniques integrated with organ-on-a-chip devices are discussed with special emphasis on maintaining the biological relevance, achieving analytical compatibility, system integration and final applicability.

Keywords: organ-on-a-chip, mass spectrometry, online analysis, electrochemical sensor, optical detector

\section{Abbreviations}

$\mathrm{CO}_{2}$ carbon dioxide

ECIS electric cell substrate impedance sensing

ESI electrospray ionization

$\mathrm{H}_{2} \mathrm{O}_{2}$ hydrogen peroxide

ICP inductively coupled plasma

IR infrared

ISFET ion sensitive field effect transistor

LAPS light addressable potentiometric sensor

MALDI matrix assisted laser desorption/ionization

MS mass spectrometry 
$\mathrm{O}_{2} \quad$ oxygen

PDMS polydimethylsiloxane

PSI paper spray ionization

SPE solid phase extraction

TEER transepithelial electrical resistance

UV-vis ultraviolet visible

VEGF vascular endothelial growth factor

\section{1. Introduction}

Reliable experimental models that mimic the function of human organs play an important role in the development of novel drugs, assessment of the toxicological effect of chemicals and monitoring the health benefits of dietary compounds. Animal models capture complex processes like absorption, distribution, metabolism and excretion of chemicals, but do not always represent human physiology adequately due to important differences between species [1]. Furthermore, worldwide scientific and socio-political organizations strive to reduce, refine and replace the use of animals for research purposes [2, 3]. Standardized, in vitro cell culture assays are currently used in early phases of drug development, food research and hazard identification of chemicals [4, 5]. However, these in vitro models lack organ specific functionality, hampering mechanism-based research needed for novel drug development and next generation risk assessment.

Recent advances in microchip- and bio-engineering enabled the development of organ-on-a-chip models, an in vitro cell culture model that includes dynamic physical and functional features of a human organ [6]. In recent years, several organ-on-a-chip models have been developed, for brain- [7, 8], lung- [9], heart- [10], kidney- [11], liver [12], skin [13], 
gut $[14,15]$ and even models that comprise multiple organ systems [16]. To establish an organ-on-a-chip model, cells are cultured within a microfluidic device simulating a tissue specific physical microenvironment. For example, Kim and colleagues have developed a human gut-on-a-chip, in which intestinal cells were grown on a permeable membrane. Interestingly, upon exposure of these cells to mechanical forces, simulating peristaltic motion, and a liquid flow, resulting in physiological relevant fluid shear stresses, tissue functionality closer resembled in vivo responses [14]. The permeable membrane separates the microfluidic channel in a top and bottom compartment which makes this model well suited for uptake studies of dietary, pharmaceutical and chemical compounds. Often multiple cell types are combined in organ-on-a-chip models, like endothelial cells [17], immune cells [18] and components of the intestinal microbiome [19], allowing mechanistic studies of more complex tissue interactions. This can be taken a step further by growing primary human cells, adults stem cells or induced pluripotent stem cells in the chip, allowing for personalized medicine testing using organ-on-a-chip technology [20].

The main scientific and technological advantages of organ-on-a-chip technology are the ability to spatiotemporally control the microenvironment and the low reagent consumption. Exploiting the unique dynamic aspects of organ-on-a-chip technology, automated online measurement of chemicals by sensors or online coupling to analytical equipment is becoming realistic. However, apart from the technical challenge to detect compounds and metabolites at very low concentrations in such miniaturized formats, the analytes will be present in cell culture medium which contains very high levels of sugar, salts, amino acids and proteins (table 1 ) that may interfere with the measurement. 
Table 1: General composition of cell culture medium

\begin{tabular}{cccc}
\hline Compound & Concentration (mg/L) & Compound & Concentration (mg/L) \\
\hline Calcium Chloride & 200 & L-Threonine & 95.2 \\
Dextrose & 4500 & L-Tryptophan & 16 \\
Ferric Nitrate & 0.1 & L-Valine & 93.6 \\
Magnesium Sulphate & 97.7 & Vitamin B5 & 4 \\
Potassium Chloride & 400 & Choline Chloride & 4 \\
Sodium Bicarbonate & 3700 & Folic Acid & 4 \\
Sodium Chloride & 6400 & I-Inositol & 7 \\
L-Arginine & 84 & Nicotinamide & 4 \\
L-Glutamine & 584 & Pyridoxine & 4 \\
Glycine & 30 & Vitamin B2 & 0.4 \\
L-Histidine & 42 & Vitamin B1 & 4 \\
L-Isoleucine & 104.8 & Phenol Red & 15 \\
L-Leucine & 104.8 & Pyruvic Acid Sodium Salt & 110 \\
L-Lysine & 146.2 & L-Tyrosine Disodium Salt & 103.7 \\
L-Methionine & 30 & L-Cystine 2HCl & 62.5 \\
L-Phenylalanine & 66 & Sodium Phosphate & 108.6 \\
L-Serine & 42 & Added Protein/Serum & variable
\end{tabular}
chip devices, excluding end-point measurements that require fixation or destruction of the cells. Here, online is defined as a direct connection between the organ-on-a-chip device and the detection method requiring no user involvement. Furthermore, in situ is defined as in close proximity to the cells. The focus is on the analysis of mammalian cell cultures rather than organ slices or single cell analysis, which have been reviewed recently [21]. Literature from 2000 till 2019 has been searched using the databases from PubMed, Scholar, Scopus and Web of Science with the following keywords: organ-on-a-chip (and organ specific variations), mass spectrometry, sensor, optical detection, in situ sensing, coupling, real-time and online analysis. The first part of the review will mainly focus on the electrochemical monitoring of the microenvironment in the organ-on-a-chip device to confirm proper biological functionality of the model, discussing cell layer integrity, mitochondrial function, extracellular oxygen and acidification. In the second part, the integration of analytical 
techniques with organ-on-a-chip devices will be addressed, focussing on optical detection, electrochemical sensing and mass spectrometric analysis of target molecules.

\section{Electrochemical monitoring of the microenvironment of organ-on-a-chip systems to}

\section{assure biological integrity}

Dynamic in vitro models like organ-on-a-chip models allow for the control of the cellular environment in great detail. However, this is only relevant if the local microenvironment can be strictly monitored [22]. Some important parameters to monitor are cell layer integrity, mitochondrial function, extracellular oxygen and acidification as they influence major chemical and biological processes in the cellular model (Fig.1). A fast and accurate detection of these parameters is a prerequisite for fast control (feedback) of the microenvironment to correct for unwanted derivations from the normal situation. Active control of the microenvironment has been extensively reviewed for organ-on-a-chip purposes [23-25]. In the following part we will discuss the integration of electrochemical sensors for organ-on-achip applications to measure: cell layer integrity, mitochondrial function, extracellular oxygen and acidification. Kieninger and colleagues [26] recently reviewed microsensors in static $2 \mathrm{D}$ and $3 \mathrm{D}$ cell cultures. Therefore, here we focus on the integration of sensors in dynamic cell based microfluidic chip systems. 

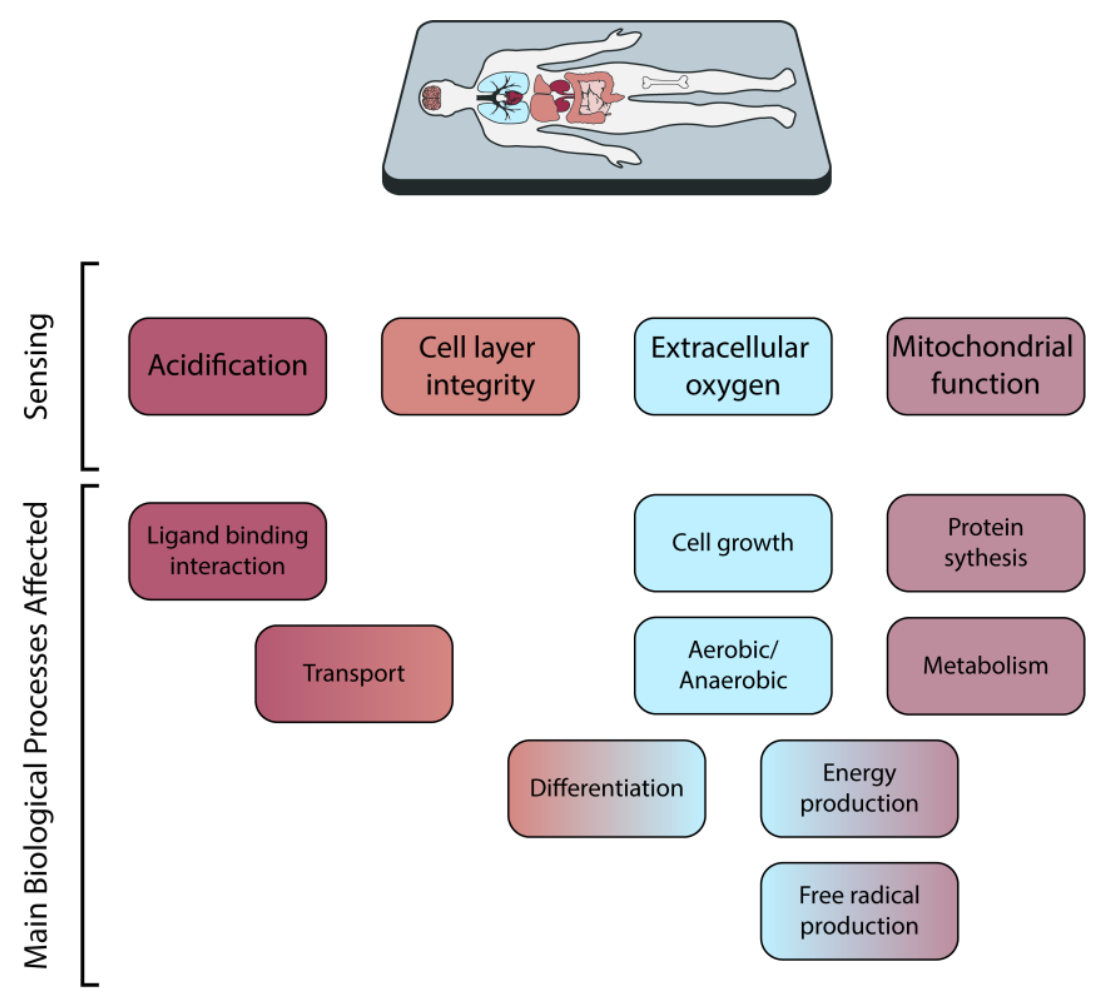

\section{Mimic biological function}

Figure 1: Simplified representation of sensing parameters in relation to cell function.

\subsection{Cell layer integrity}

Transepithelial electrical resistance (TEER) is a measure for the barrier integrity of epithelial and endothelial cell layers [27]. In a classical experimental setup, TEER measurements are performed before and after exposure to compounds as barrier integrity quality control. Alternatively, TEER data is used as read out of diseased "leaky" models, or as a marker of toxicity upon exposure to a compound. TEER measurements are non-invasive, label-free and performed in real-time. In the conventional in vitro transwell system (Fig.2), TEER is measured by manually submerging (silver) electrodes in the top and bottom compartment of the transwell insert. The electric resistance is measured over the cell layer, which increases with an increasing tightness of the cell layer [27]. However, manually submerging these electrodes in the confined closed areas in organ-on-a-chip devices is rather tricky. The cell culture area in microfluidic devices is generally much smaller compared to transwell systems 
which makes positioning of the electrodes in close proximity of the cells, crucial for a stable measurement. Attachment of the electrodes to the device itself would eliminate the noise generated by the movement of the electrode by the user.

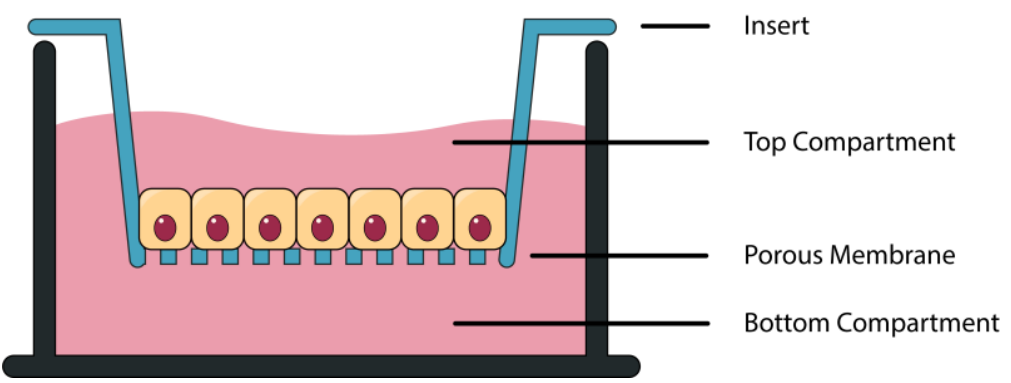

Figure 2: Conventional transwell insert

TEER measurement electrodes have been incorporated in organ-on-a-chip models like the blood-brain-barrier- [7, 8], gut-, lung- [28, 29], heart- [30] and skin-on-a-chip [13]. For example, a chip consisting of two polydimethylsiloxane (PDMS) channels, separated by a membrane, was closed on either side by glass slides. On these glass slides, $25 \mathrm{~nm}$ thick transparent gold electrodes were sputter-coated along the full length of the channel and attached to thin copper wires, which were connected to a multimeter for TEER analysis [28]. Currently, TEER electrodes are not attached to flexible surfaces that are used for stretching the cell layer, for instance in lung-, or gut-on-a-chip systems. Such sensor integration for flexible surfaces has been shown for other purposes [31, 32]. Possible solutions for TEER analysis lie in further miniaturization of the electrodes and synthesizing the electrode from a flexible material. Moving away from static transwell barrier models, raises the question which TEER values in organ-on-a-chip devices are considered as indicative of a mature monolayer barrier. Data from literature has shown that TEER values in microfluidic chips are rather different compared to values measured in transwells using the same cell type [27]. It has been reported that this is most likely due to different geometries and materials of microfluidic chips, compared to a traditional transwell system rather than being a result of 
biological differences in chip systems. To account for these differences a mathematical model was developed [33]. In this model, several parameters like channel height and width, membrane length, conductivity of the cell culture medium and resistance of the membrane material are taken into account [33]. Clearly, integration of electrodes for TEER measurements adds to the complexity of fabrication and use of organ-on-a-chip devices resulting in higher costs. But barrier models on chip greatly benefit from the incorporation of TEER electrodes to be able to reliably measure the integrity of epithelial and endothelial cell layers.

Electric cell substrate impedance sensing (ECIS) is another sensing technique for cellular monolayer integrity that is integrated in organs-on-a-chip devices. This method is not only used to assess barrier integrity, but also is a well-known non-invasive method to measure cytotoxicity, cell proliferation or wound healing properties [34]. With ECIS, cells are grown on a gold electrode, the impedance of the electrode is measured at one or more frequencies versus time. As cell membranes have insulating properties the more cells that are present, the higher the impedance measurement. ECIS has successfully been integrated in different types of organ-on-a-chip models like a hydrogel based model [35] and PDMS based models [36, 37].

A general problem for all types of in situ electrochemical sensors is the continuous exposure to cell culture medium, which can result in fouling of the electrode. Frequent cleaning, shorter experiments or the incorporation of antifouling layers would minimize the effect of fouling on the electrode [38].

\subsection{Mitochondrial function}

Mitochondria are the powerhouses of the cell, producing adenosine triphosphate through 
the respiration chain. Monitoring mitochondrial activity is essential for evaluating the energy demand of the cell and is commonly used to monitor the viability of cells. Measurement of glucose and lactate levels in the surrounding cell culture medium is a frequently used procedure to analyse mitochondrial activity in organ-on-a-chip models [39-42]. The production of lactate, parallel to the decline of glucose through glycolysis, is a sign of mitochondrial dysfunction. Bavli and colleagues measured glucose and lactate levels in a liver-on-a-chip device, in which a sensor unit was attached downstream of the microfluidic device [43]. The sensor included membrane embedded glucose and lactate oxidase and platinum electrodes, which were stable for 24 hours of measurements. Every hour, cell culture medium from the liver chip was introduced to the sensor unit where both glucose and lactate were oxidized under the formation of hydrogen peroxide $\left(\mathrm{H}_{2} \mathrm{O}_{2}\right)$, the latter being measured using the platinum electrode. The disadvantage of this sensor is that as a result of the production of $\mathrm{H}_{2} \mathrm{O}_{2}$ and the use of oxygen $\left(\mathrm{O}_{2}\right)$ for this process, the sensor unit must be separated from the cells [43]. In addition, the measurements are not continuous and fouling issues apply again. Fouling issues are circumvented by shorter experiments, which is not ideal for chronic biological experiments. Nevertheless, monitoring glucose and lactate levels in organ-on-a-chip device is important for the evaluation of proper mitochondrial activity.

\subsection{Extracellular oxygen}

Oxygen is crucial for the conversion of nutrients into energy within the cell. Reduced levels of oxygen result in anaerobic cell respiration, causing less efficient energy transfer, which can only be sustained for a limited time. During cellular respiration carbon dioxide $\left(\mathrm{CO}_{2}\right)$ is produced, resulting in the acidification of the cell culture medium if not properly buffered. Traditional in vitro cell culture models are grown in a culture plate or flask and are placed inside an incubator where $\mathrm{CO}_{2}$ levels are controlled. Culture plates and flasks are open 
systems and $\mathrm{O}_{2}$ and $\mathrm{CO}_{2}$ exchange takes place inside the incubator [44]. Gas exchange is rather different in organ-on-a-chips, as these are commonly closed systems. Aspects to consider are chip material, smaller media-to-cell volume and ambient environment. Nowadays, most microfluidic chips are made of PDMS, which has a high gas diffusion coefficient. This allows for sufficient exchange of $\mathrm{O}_{2}$ and $\mathrm{CO}_{2}$, when the PDMS layer is thin enough $(\sim 100 \mu \mathrm{m})[45]$.

Alternative microfluidic chip materials are being studied because of some important disadvantages of PDMS from a biological perspective. A well-known disadvantage of PDMS is the high likelihood of absorption of hydrophobic compounds to PDMS, even though several coating procedures have been proposed to avoid this [46]. Much less-known, but very relevant in terms of potentially limiting the online coupling to sensitive analytical detection systems is the leaching of uncross-linked oligomers and polymer additives into the media [46]. Several other materials like, glass [47] and polycarbonate [48] are used for chip fabrication, but are less permeable to gasses. All these factors influence $\mathrm{O}_{2}$ and $\mathrm{CO}_{2}$ exchange in organ-on-a-chip devices, which is why integration of oxygen sensors in chip systems is of great importance. Incorporation of oxygen sensors also allows for studying the respiration chain by precise monitoring of energy production. Lastly, the incorporation of oxygen sensors is pivotal for the development of advanced gut-on-a-chip models. In these models tight monitoring (and adjustments) of low oxygen levels are required to maintain anaerobic growth conditions needed for the inclusion of a human relevant intestinal microbiome $[19,49]$.

The most often applied approach for sensing oxygen is by the use of metal electrodes, like silver, gold or platinum. Oxygen levels are measured based on the 
amperometric reduction of dissolved oxygen [12, 39, 40, 47, 50, 51]. A major disadvantage of using metal electrodes in microfluidic systems however is the reduction of $\mathrm{O}_{2}$ to $\mathrm{H}_{2} \mathrm{O}_{2}$ during the measurement. This makes the sensor unsuitable for placement in close contact with the cells. To sum up, oxygen levels in organ-on-a-chip devices can be variable depending on chip material and ambient environment, therefore incorporation of oxygen sensors on chip is crucial for maintaining a biological relevant microenvironment.

\subsection{Acidification}

Mammalian cells function best at a neutral $\mathrm{pH}$. As mentioned in paragraph 2.3, the improper exchange of $\mathrm{CO}_{2}$, can result in an undesirable acidification of the extracellular environment. To keep track of the cellular environment, most cell culture media contain phenol red, as a $\mathrm{pH}$ indicator. However, due to the small volumes in organ-on-a-chip devices colorimetric changes are difficult to observe visually. Integration of $\mathrm{pH}$ sensors in organ-on-a-chip systems would allow for direct feedback and control measurements to prevent undesirable $\mathrm{pH}$ fluctuations. Zhang and colleagues described a liver-heart chip model with an incorporated $\mathrm{pH}$ sensor that detected changes in absorbance of phenol red [52]. More widespread are silicon based chemical sensors, like the light-addressable potentiometric sensors (LAPS) $[53,54]$. LAPS in conventional cell culture applications are constructed of silicon chips that are placed at the bottom of a cell culture chamber (Fig.3). 


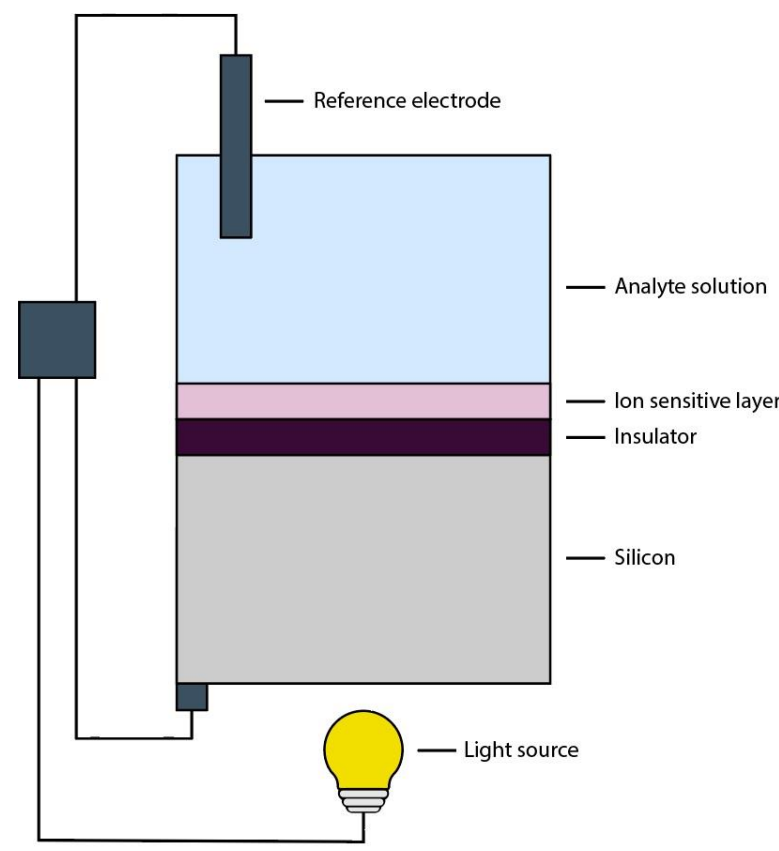

Figure 3: Schematic representation of LAPS

The silicon chip has an insulating layer and an ion sensitive layer consisting of silicon oxide and silicon nitride. The ion sensitive layer interacts with the protons within the cell culture medium, affecting the surface potential of the layer. The surface charge of the ion sensitive

Even though both LAPS and ISFET are sensitive $\mathrm{pH}$ sensors, LAPS sensors are preferred 
the electrode has a major effect on the sensitivity of the electrode. In current literature LAPS and ISFET sensors are regularly cleaned, sterilized and used for short experiments to mitigate the impact of fouling.

In conclusion, many different integrated electrochemical sensors have been developed to monitor the cellular microenvironment in organ-on-a-chip devices over the past couple of years. Optical sensing techniques, like photoacoustic imaging and luminescence detection have been or have the potential to be incorporated on chip as well, however they require a dye or labelled substrate for visualisation [60-62]. Electrochemical sensors do not have this drawback, which explains the more widespread use of these type of sensors. Ideally, all parameter measurements, pH, TEER, glucose, lactate and oxygen, are combined to establish a broader picture of cell functioning in homeostasis and under stress. Future work should concentrate on the combination of different robust sensors in one user friendly format to study various biological processes within organ-on-a-chip devices.

\section{Integration of analytical techniques for target substance detection}

Organ-on-a-chip devices have been integrated with multiple analytical techniques, like optical spectroscopy, electrochemical sensors and mass spectrometry. These integrations widen the applicability of organ-on-a-chip models for drug uptake and dietary studies and unravelling biological processes. In the following part we will discuss the different integrations and the major challenges that relate to sensitivity and selectivity of detection in organ-on-a-chip systems in the highly complex and abundant cell culture medium (table 1).

\subsection{Optical spectroscopy detection of target analytes}

Optical detection instruments are abundant in most laboratories and ultraviolet visible (UV-

Vis) spectroscopy, infrared (IR) spectroscopy, luminescence, and microscopy versions 
thereof, have been applied for the detection of a wide range of analytes in organ-on-a-chip devices. Integration of a spectrophotometric detection system in an organ-on-a-chip model has been shown in a membrane based kidney-on-a-chip. The chip was connected with two flow channels, one for either side of the membrane. Each channel was directed through quartz cuvettes allowing real-time analysis of caffeine and vitamin B12 permeability $[11,63]$. Another label-free option reported is IR spectroscopy. The main problem with IR and organon-a-chip technology is the liquid barrier on top of the cells since IR absorption by water will interfere with the signal. However, Loutherback and colleagues came up with a solution for this problem [48]. They created a chip containing two channels, separated by a gold coated porous membrane on which neuronal cells were grown. During measurements little to no liquid was present on top of the cells, but a flow of $100 \mathrm{~nL} / \mathrm{min}$ was maintained to the lower channel of the device to still provide the cells with the right nutrients and prevent them from drying. Different regions as a function of cell stress could be visualised within the cell culture on the basis of the peak intensity of vibrational modes of $\mathrm{C}-\mathrm{O}-\mathrm{C}, \mathrm{C}-\mathrm{O}-\mathrm{P}$ and $\mathrm{C}-\mathrm{O}$ stretching of glycogen/glycoprotein and they demonstrated continuous measurements for up till a week [48]. Despite this achievement, the application seems more suited for skin- or lung-on-a-chip that grow at the air liquid interface.

Optical biosensors are popular techniques to detect target peptides and proteins in organ-on-a-chip models. Two types of biorecognition elements are found in organ-on-a-chip integrations namely, aptamers like deoxyribonucleic acid or ribonucleic acid $[64,65]$, or antibodies $[52,66]$. In organ-on-a-chip models, the biggest concern for the applicability of integrated biosensors is the overabundance of nonspecific proteins compared with the trace levels of the analyte of interest. A nanoplasmonic platform that employs an antibody based biosensor was integrated with an organ-on-a-chip to quantitatively determine cellular 
cytokine release in real-time and label-free [67]. The platform consisted of two parts: a cellular compartment and an optical detection compartment where secreted cytokines were detected (Fig.4). The optical detection module contained three inline nanohole arrays, one as negative control and the other two functionalized with a specific antibody against the cytokine of interest. A beam of broadband light was directed onto the nanohole array and the transmitted light was measured by a spectrometer. Binding of the cytokine to the antibody caused a detectable wavelength shift of the transmitted light [67]. Besides antibodies, aptamer based biosensors are also used in organ-on-a-chip devices. Claimed advantages of aptamers compared to antibodies are: better binding capacities to any given target and highly reproducible animal free production methods with high purity [68]. However, the presence of deoxyribonuclease and ribonuclease enzymes in biological samples makes aptamers susceptible to degradation. An example of an aptamer biosensor is the integrated vascular endothelial growth factor (VEGF) aptamer biosensor for cervical cancer cells on chip. The biosensor consisted of a functional nucleic acid, designed to bind to VEGF and was immobilized onto the surface of the chip. The aptamer was coupled to a Gquadruplex DNazyme, acid, hemin and peroxide system which upon binding of VEGF catalysed the reaction resulting in a blue-green colour that was analysed by Vis spectroscopy [65].

Fluorescent dyes have been used to visualize target molecules in an organ-on-a-chip by fluorescence microscopy [8, 19, 47]. Alternatively, fibre optics [69] and even smartphones [70] have been exploited as miniaturized fluorescent detectors for organ-on-a-chip devices. The fluorescence microscope developed by Cho et al. [70] consisted of three white light emitting diodes, two optical filters and an objective lens. Images were taken with the smartphone and analysed separately on a computer. They demonstrated its use in 
combination with a kidney-on-a-chip device. The cells on the chip were exposed to a specific kidney toxin, which induced the release of a brush border enzyme. Subsequently, an antibody, conjugated to a fluorescent nanoparticle label, bound to the enzyme and the fluorescence signal was detected using the smartphone microscope. Using a smartphone as a read-out simplifies and decreases the cost of analysis, however it may also compromise the sensitivity in comparison to a conventional fluorescence microscope.

a. Cell Module

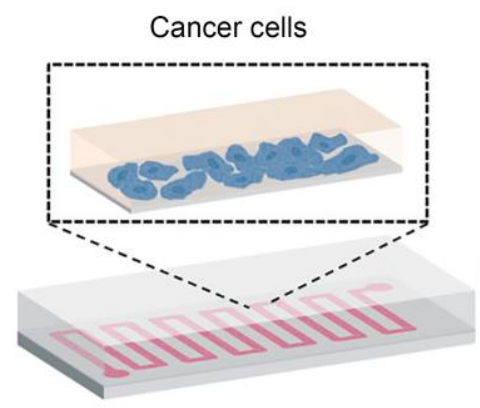

b. Cell Module

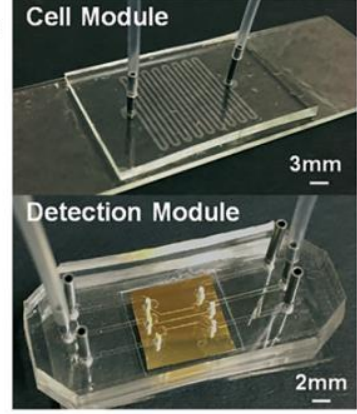

Detection Module

Broadband illumination
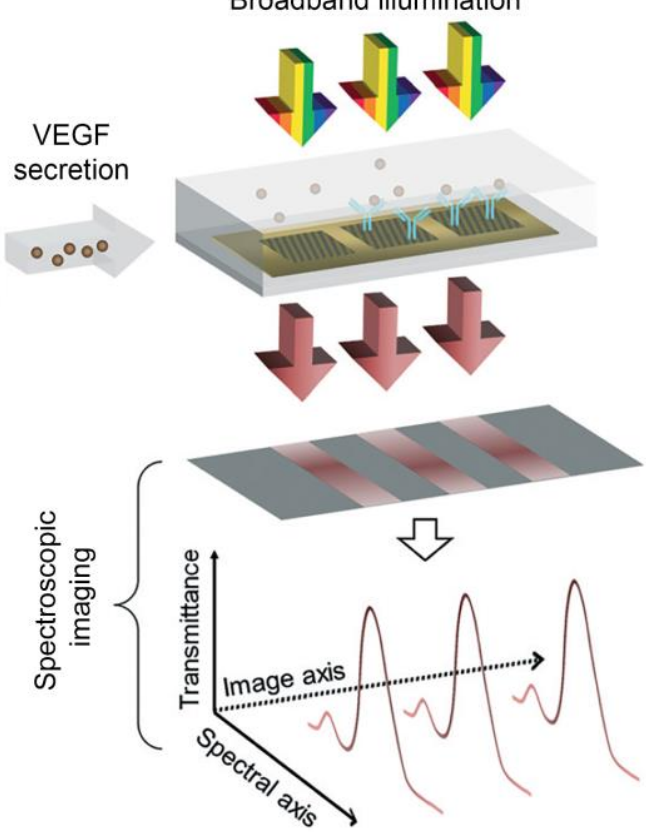

Figure 4: (a) Schematic representation of a microfluidic integrated biosensor for real-time cytokine analysis. (b) Photo of cell culture module and detection module. Reproduced from Ref. [67] with permission of The Royal Society of Chemistry.

Integration of optical detection methods with organ-on-a-chip devices is one of the

few in situ techniques that allows for long term analysis, because cells are generally not

disrupted during the measurements. However, some integrative techniques require a

labelled substrate to visualize the compound in the cell culture matrix.

\subsection{Electrochemical detection of target analytes}

Amperometric models for the detection of target analytes have been proposed in several 
organ-on-a-chip systems [71, 72], showing an alternative application of electrochemical sensors next to the previously discussed application as tools to monitor the extracellular microenvironment. An example of an electrochemical sensor based on amperometric reduction was described by Li et al. [72]. They designed a PDMS microchip with an integrated electrochemical sensor measuring a redox reaction at the surface of a platinum electrode at the bottom of the channel. In the chip, immobilized neuronal cells were grown and subsequently stimulated with calcium ions to induce the release of dopamine. Using the platinum electrode, the oxidation and release of catecholamine (dopamine/epinephrine) could be measured in the micromolar range. The disadvantage of this method is that no distinction between dopamine and epinephrine could be made, since they have the same redox potential [72]. Another method described is an impedance spectroscopy antibody biosensor platform with a built-in regeneration function to prevent sensor saturation [66]. A series of on chip pressure driven microfluidic valves allowed for the regeneration of the sensor and detection without manual interference, thus decreasing the possibility of human error. Regeneration of the sensor was established by flowing a cleaning solution over the chip at a high speed and an electrical sweep. To demonstrate robustness of the method they compared the results from the impedance spectroscopy sensor with a conventional enzyme linked immunosorbent assay which showed similar sensitivity [66]. The biggest challenge for electrochemical detection is fouling of the electrode surface, decreasing the overall sensitivity and robustness of the sensor. The integration of cleaning steps or a selective antifouling layer would greatly improve the usability of electrochemical detection of target analytes in microfluidic chips [38].

\subsection{Mass Spectrometric detection of target analytes}

Organ-on-a-chip devices accommodate minimal amounts of analytes in a highly complex 
cellular environment, which puts high demands on the analytical instrumentation in terms of sensitivity and sample preparation. Mass spectrometry (MS) is a label-free and multi analyte detection technique that meets these challenges provided that ion suppression due to the cellular environment can be overcome. The integration of a microfluidic chip to MS has been reviewed recently [73-76]; here we focus on the online analysis of organ-on-a-chip models with MS.

\subsubsection{Electrospray ionization MS}

Electrospray ionization (ESI) is ideal for interfacing with dynamic organ-on-a-chip devices, as ionization of target compounds occurs in the liquid phase. Clearly, a major challenge is the complex mixture with high concentrations of sugars, salts and proteins in the cell culture medium (table 1) causing severe ion suppression thereby compromising the detection of the analyte of interest. A solution is the integration of a solid phase extraction (SPE) column, either incorporated on the same chip [77] or coupled to the chip [78]. On chip SPE coupled to ESI-MS has been studied by the group of Jin-Ming Lin [77, 79-85]. For example, a microfluidic system was developed to characterize curcumin permeability across an intestinal epithelial layer (Fig.5). The system consists of two parts, part one a membrane based cell culture chip, where intestinal cells were cultured on a permeable membrane separating a top and bottom chamber. The bottom chamber of the membrane was connected to the second part of the system, a chip containing a micro-SPE column. The SPE column captured curcumin that permeated through the cell layer and was washed offline with a water-methanol mixture to remove any unwanted sugars and salts. Then, the microSPE chip was connected to the ESI-MS via fused silica capillaries for the detection of curcumin [77]. A major drawback of this system is the offline washing step of the SPE column which compromises the overall online nature and time resolution of the system. Similarly, 
the group of Jin-Ming Lin was able to couple several other organs-on-a-chip systems to ESIMS, such as neurons-[81, 82, 84], liver-[79, 83, 85] and lung-on-a-chip [80]. Others used separate SPE columns coupled to their chip to capture their analyte of interest. For instance, Dugan et al. [86] developed a chip to analyse the release of nonesterified fatty acids from fat tissue cells. An on-chip sample loop collected the released fatty acids and was subsequently eluted by an on-chip automated valve system to a separate SPE column [86]. Another exciting example is a system using a series of three switching valves to measure the effect of cocaine on cells of the immune system in near real-time. This system included two loops for continuous sample collection and SPE columns for desalting [78]. The advantage of sample preparation in a column isolated from the chip is that commercially SPE columns can be applied and elution and wash steps can be easily automated.

Maintaining a stable cell temperature of $37^{\circ} \mathrm{C}$ and controlling $\mathrm{O}_{2} / \mathrm{CO}_{2}$ gas flows together with online analysis is a serious challenge of organ-on-a-chip systems integrated with large footprint analytical equipment, such as MS. In table 2 an overview is given of organ-on-a-chip systems hyphenated with mass spectrometry detection evaluating the biological relevance and online nature of the systems. From this table it is clearly shown that either the biological relevance of the organ-on-a-chip mimic or the online analysis of the system is significantly compromised. 

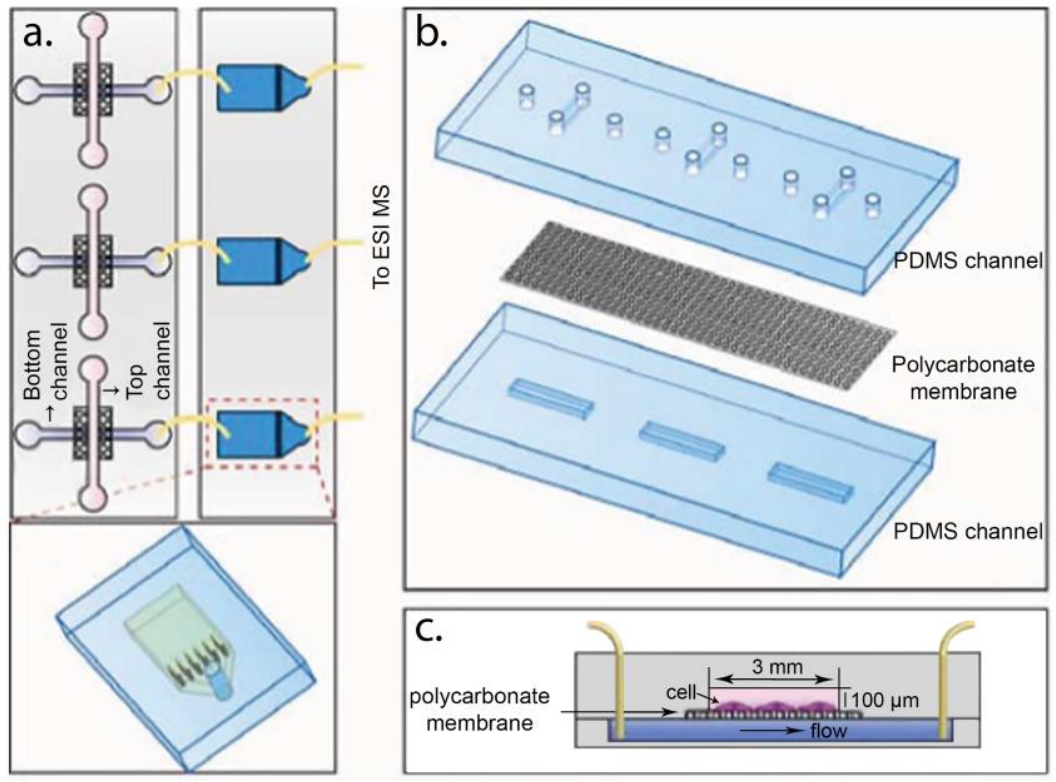

Figure 5: (a) Schematic representation of microfluidic device for cell culture and ESI-MS detection. (b) The three layers of the membrane based cell culture chip. (c) Side view cell culture chip, not to scale. Reproduced from Ref. [77] with permission of The Royal Society of Chemistry.

\subsubsection{Ambient ionization MS}

Ambient ionization MS was pioneered by Cooks and Cody by the invention of desorption electrospray ionization [87] and direct analysis in real-time [88]. Nowadays, a plethora of related designs have become available [89]. Paper spray ionization (PSI) is an ambient ionization technique where the sample is deposited on a triangular piece of paper in front of the MS entrance [90]. The main advantages of using paper includes low costs, wide availability and the possibility of chemical modification of the paper [91]. Two types of integrations are reported in literature coupling PSI to organ-on-a-chip systems. The first type is a microdialysis PSI-MS system which monitored the glucose concentration in the media [92]. Human liver cells were stimulated with insulin and the decrease in glucose levels within the system was followed [92]. However, the cells were grown in a static petri dish, opposed to a dynamic microfluidic system. Later the same group developed a four channel microfluidic chip in which they monitored lactate production of normal cells versus tumour 
cells [93]. The second type of integration is a system in which cells were directly grown on

the paper substrate. However, conventional chromatography paper for cell culture has its drawbacks, mainly in mechanically supporting the cells [94]. Both glass and polycarbonate substrates have been used to provide a scaffold for cell culture and direct PSI-MS analysis $[95,96]$. However, in both cases, cells are lysed by isopropanol for PSI-MS analysis making it an end-point measurement. PSI is in theory a well suited method for fast screening of a cellbased microfluidic chip. Nevertheless, temperature and $\mathrm{CO}_{2}$ control remain a big challenge for any method operated in an open ambient environment.

Table 2: Overview of organ-on-a-chip systems hyphenated with mass spectrometry. Biological relevance and full online analysis were evaluated.

\begin{tabular}{|c|c|c|c|c|c|c|c|c|}
\hline \multirow[b]{2}{*}{$\begin{array}{l}\text { Detection } \\
\text { Method }\end{array}$} & \multirow[b]{2}{*}{ Cell Model } & \multicolumn{4}{|c|}{ Biological relevance } & \multirow{2}{*}{$\begin{array}{c}\text { Fully Online } \\
\text { Analysis } \\
\text { (yes/no) }\end{array}$} & \multirow[b]{2}{*}{ Analyte } & \multirow[b]{2}{*}{ Ref } \\
\hline & & Temp & $\mathrm{CO}_{2}$ & $\begin{array}{c}\text { Cell } \\
\text { Viability }\end{array}$ & $\begin{array}{c}\text { Barrier } \\
\text { Integrity }\end{array}$ & & & \\
\hline ESI-MS & $\begin{array}{l}\text { Caco-2 } \\
\text { cells }\end{array}$ & + & + & + & + & no & Curcumin & [77] \\
\hline ESI-MS & Jurkat cells & + & + & + & n.a. & yes & Cocaine & [78] \\
\hline ESI-MS & A549 cells & + & + & + & n.a. & no & Vitamin E & [80] \\
\hline ESI-MS & PC12 cells & + & + & + & n.a. & no & Glutamate & [81] \\
\hline ESI-MS & $\begin{array}{l}\text { PC12 and } \\
\text { GH3 cells }\end{array}$ & + & + & - & n.a. & no & $\begin{array}{c}\text { Growth } \\
\text { hormone }\end{array}$ & {$[82]$} \\
\hline ESI-MS & $\begin{array}{l}\text { HepG2 } \\
\text { cells }\end{array}$ & + & + & + & n.a. & no & Acetaminophen & [83] \\
\hline ESI-MS & $\begin{array}{l}293 \text { and L- } \\
02 \text { cells }\end{array}$ & - & - & + & n.a. & no & $\begin{array}{c}\text { Epinephrine and } \\
\text { glucose }\end{array}$ & [84] \\
\hline ESI-MS & $\begin{array}{l}\text { HepG2 and } \\
\text { MCF-7 cells }\end{array}$ & + & + & + & n.a. & no & $\begin{array}{l}\text { Capecitabine } \\
\text { metabolites }\end{array}$ & [85] \\
\hline ESI-MS & 3T3-L1 & - & - & - & n.a. & yes & $\begin{array}{l}\text { Non-esterified } \\
\text { fatty acids }\end{array}$ & [86] \\
\hline PSI-MS & $\begin{array}{l}\text { HepG2 and } \\
\text { L-02 cells }\end{array}$ & + & + & n.a. & n.a. & yes & Glucose & [92] \\
\hline PSI-MS & $\begin{array}{l}\text { A549, L-02 } \\
\text { and MCF-7 } \\
\text { cells }\end{array}$ & + & + & n.r. & n.a. & yes & Lactate & [93] \\
\hline
\end{tabular}

\subsubsection{Other MS options}

The majority of MS coupling methods to organs-on-a-chip considered the coupling to ESI-MS and PSI-MS rather than other ionization techniques. Two alternative types of ionization 
techniques that would be beneficial in the field of organ-on-a-chip are inductively coupled plasma (ICP) MS and matrix assisted laser desorption/ionization (MALDI) MS. ICP-MS would be beneficial for the analysis of metal ions or particles frequently present in food products, as an additive, or for pharmaceutical purposes [97]. MALDI-MS imaging has been used for the analysis of neuropeptide release from Aplysia neuronal cells on chip [98], but further studies are limited.

Organ-on-a-chip models have been integrated with several different analytical techniques for the detection of target analytes, all dealing with issues of sensitivity and selectivity. The most promising integration in terms of sensitivity, selectivity and multi analyte detection seems to be ESI-MS with the integration of a SPE column to get rid of the interference of the cell culture medium. However, when truly in situ analysis is required for a specific biological application, optical or electrochemical sensing techniques provide a simpler coupling solution.

\section{Conclusions}

Recent advances in integrated analytical techniques with organ-on-a-chip devices were discussed. Main advantages of these integrations are reduction of (bio)reagents, automation allowing unattended prolonged experiments and real-time analytical data for feedback on nutrient composition and detection of target analytes and metabolites thereof. Organ-on-achip devices are living cellular systems, therefore careful real-time monitoring of the functioning of the cells is crucial to ensure the biological relevance of the micro tissue. Main challenges for integrated analytical techniques comprise sensitivity, selectivity, robustness, user friendliness and multi analyte detection. In situ optical and/or electrochemical sensors are easy to use analytical devices and small enough to be placed inside a gas and 
temperature controlled incubator. Issues that remain to be solved however are, lower sensitivity compared to conventional benchtop analytical equipment, susceptibility to fouling and measurement of only one (or a limited number) of parameters at a time. Future analytical solutions for online organ-on-a-chip systems can be found in the design of multisensor platforms. Surfaces of such multisensors should have tailor made antifouling layers to mitigate nonspecific binding and the sensors should provide active feedback control loops, thus ensuring a stable microenvironment for biological relevant in vitro experiments.

Online coupling to high-end instrumentation such as a mass spectrometer is another crucial future development. That would enable the semi-continuous identification and quantification of multiple target analytes, and (un)expected metabolites thereof, in a small sample volume with high sensitivity. Continuous online mass spectrometric detection of organ-on-a-chip systems is currently not feasible, due to the presence of high levels of interfering substances in the cell culture medium that require the incorporation of SPE columns and a wash step to prevent ionization suppression. In recent interfacing designs, the organ-on-a-chip device is generally placed outside the gas and temperature controlled incubator to allow interfacing with a mass spectrometer. Obviously, this is still a serious drawback as it compromises a biological accurate environment.

$$
\text { development trials and may, in the far future, even function as a diagnostic tool for }
$$
personalized medicine. Analytical techniques connected or included in the organ-on-a-chip must enable these developments. Therefore, future advancements should aim to create total analysis systems for organ-on-a-chip devices (Fig. 6), ultimately making the systems 
cheaper, more robust and more user friendly. To achieve such a system future work should

consider the following aspects and solve current problems. Firstly, advanced self-regulating organ-on-a-chip systems having sensor-based active feedback control regulating nutrient demand. Secondly, creating robust electrochemical sensors by solving fouling issues with antifouling layers based on covalent surface chemistry. Where necessary, these electrode materials may be adapted to mechanical stretching organ-on-a-chip systems, for example through the development of polymeric electrode materials. A last remaining issue is the formation of hydrogen peroxide while sensing oxygen or glucose/lactate, which might cause biological damage to the cells grown in the organ-on-a-chip device. We envisage the further integration of organ-on-a-chip systems with miniaturized analytical equipment in order to provide continuous read outs of target analytes and metabolites thereof. Eventually this will yield online systems that provide continuous online data and mimic real life in vivo biological processes. This would greatly advance the widespread use of organ-on-a-chip approaches in research and development of novel drugs, assessment of toxicological effect of chemicals and monitoring of health benefits of dietary compounds.

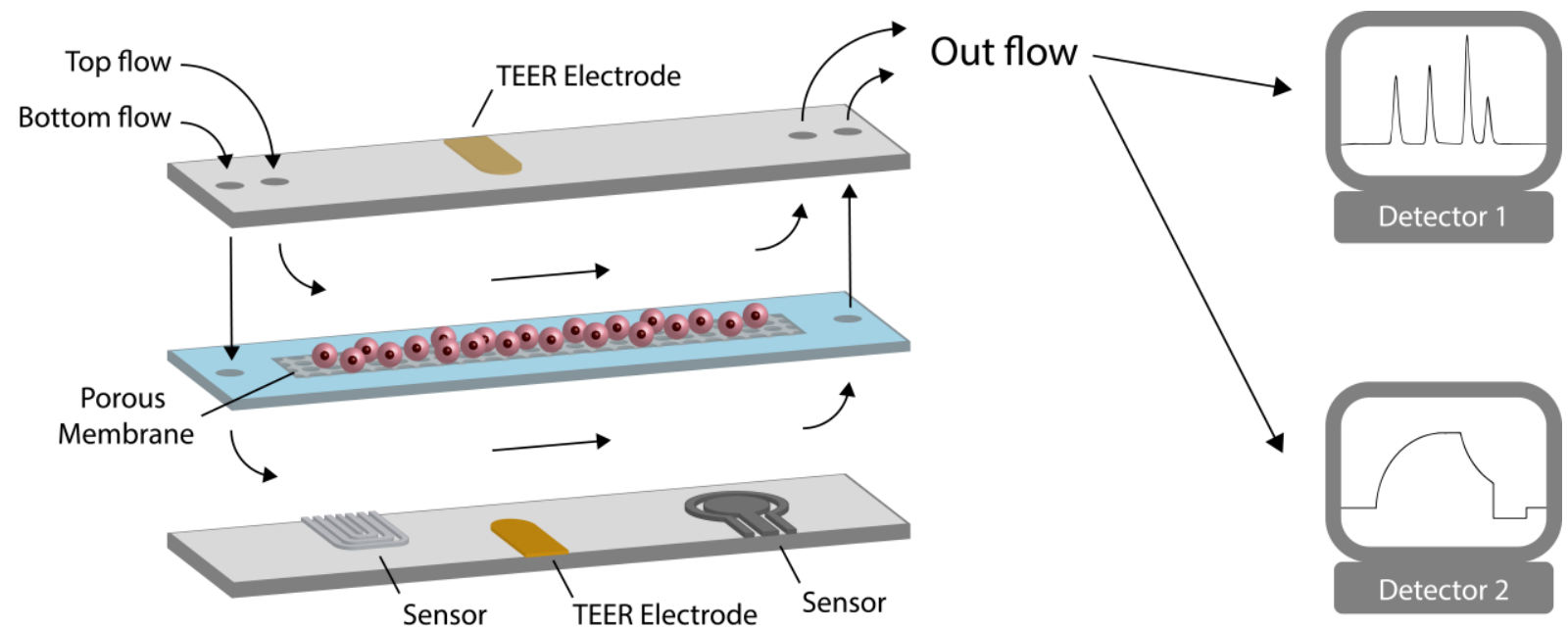

Figure 6: Advanced organ-on-a-chip model with several integrated sensors for monitoring and feedback of biological integrity and hyphenated to one or more online detectors for detection of target analytes and metabolites thereof. 
(NWO) in the framework of the Technology Area PTA-COAST3 (project nr. 053.21.116) of the

FrieslandCampina, Micronit Microtechnologies, Galapagos and Europroxima as partners.

\section{References}

[1] N. Shanks, R. Greek, J. Greek, Philos Ethics Humanit Med 4 (2009) 2.

[2] Directive 2010/63/EU of the European Parliament and of the Council of 22 September 2010 on the protection of animals used for scientific purposes, Off J Eur Commun. 2010, pp. 33.

[3] P.A. Ward, Blanchard, R. J., \& Bolivar, V, Recognition and Alleviation of Distress in Laboratory Animals, National Academies Press, Washington (DC), 2008.

[4] A. Carere, A. Stammati, F. Zucco, Toxicol Lett 127 (2002) 153.

[5] N. Fotaki, Expert Rev Clin Pharmacol 2 (2009) 195.

[6] U. Marx, H. Walles, S. Hoffmann, G. Lindner, R. Horland, F. Sonntag, U. Klotzbach, D. Sakharov, A. Tonevitsky, R. Lauster, Atla-Altern Lab Anim 40 (2012) 235.

[7] L.M. Griep, F. Wolbers, B. de Wagenaar, P.M. ter Braak, B.B. Weksler, I.A. Romero, P.O. Couraud, I. Vermes, A.D. van der Meer, A. van den Berg, Biomed Microdevices 15 (2013) 145.

[8] R. Booth, H. Kim, Lab Chip 12 (2012) 1784.

[9] D. Huh, B.D. Matthews, A. Mammoto, M. Montoya-Zavala, H.Y. Hsin, D.E. Ingber, Science 328 (2010) 1662.

[10] A. Grosberg, P.W. Alford, M.L. McCain, K.K. Parker, Lab Chip 11 (2011) 4165.

[11] C. Desrousseaux, J.M. Prot, M. Dufresne, P. Paullier, E. Leclerc, Sensor Actuat B-Chem 174 (2012)

465.

[12] A. Moya, M. Ortega-Ribera, X. Guimerà, E. Sowade, M. Zea, X. Illa, E. Ramon, R. Villa, J. GraciaSancho, G. Gabriel, Lab Chip 18 (2018) 2023.

[13] F.A. Alexander, S. Eggert, J. Wiest, Genes 9 (2018) 114.

[14] H.J. Kim, D. Huh, G. Hamilton, D.E. Ingber, Lab Chip 12 (2012) 2165.

[15] K. Kulthong, L. Duivenvoorde, B.Z. Mizera, D. Rijkers, G. ten Dam, G. Oegema, T. Puzyn, H. Bouwmeester, M. van der Zande, RSC Adv 8 (2018) 32440.

[16] J.H. Sung, Y.I. Wang, N. Narasimhan Sriram, M. Jackson, C. Long, J.J. Hickman, M.L. Shuler, Anal Chem 91 (2019) 330.

[17] L.J. Chen, S. Ito, H. Kai, K. Nagamine, N. Nagai, M. Nishizawa, T. Abe, H. Kaji, Sci Rep 7 (2017) 3538.

[18] D. Irimia, X. Wang, Trends Biotechnol 36 (2018) 923.

[19] H.J. Kim, H. Li, J.J. Collins, D.E. Ingber, P Natl Acad Sci USA 113 (2016) E7.

[20] S. Ahadian, R. Civitarese, D. Bannerman, M.H. Mohammadi, R. Lu, E. Wang, L. Davenport-Huyer, B. Lai, B. Zhang, Y. Zhao, S. Mandla, A. Korolj, M. Radisic, Adv Healthc Mater 7 (2018) 1700506.

[21] A. Reece, B.Z. Xia, Z.L. Jiang, B. Noren, R. McBride, J. Oakey, Curr Opin Biotech 40 (2016) 90.

[22] S. Varma, J. Voldman, Lab Chip 18 (2018) 3333.

[23] H. Kamble, M.J. Barton, M. Jun, S. Park, N.T. Nguyen, Lab Chip 16 (2016) 3193.

[24] A.G.G. Toh, Z.P. Wang, C. Yang, N.T. Nguyen, Microfluid Nanofluid 16 (2014) 1.

[25] E.E. Ergir, B. Bachmann, H. Redl, G. Forte, P. Ertl, Front Physiol 9 (2018) 1417.

[26] J. Kieninger, A. Weltin, H. Flamm, G.A. Urban, Lab Chip 18 (2018) 1274.

[27] B. Srinivasan, A.R. Kolli, M.B. Esch, H.E. Abaci, M.L. Shuler, J.J. Hickman, Jala 20 (2015) 107.

[28] F.R. Walter, S. Valkai, A. Kincses, A. Petneházi, T. Czeller, S. Veszelka, P. Ormos, M.A. Deli, A. Der, Sensor Actuat B-Chem 222 (2016) 1209.

[29] O.Y.F. Henry, R. Villenave, M.J. Cronce, W.D. Leineweber, M.A. Benz, D.E. Ingber, Lab Chip 17 (2017) 2264.

[30] B.M. Maoz, A. Herland, O.Y.F. Henry, W.D. Leineweber, M. Yadid, J. Doyle, R. Mannix, V.J. Kujala, E.A. FitzGerald, K.K. Parker, D.E. Ingber, Lab Chip 17 (2017) 2294.

[31] S. Zhao, J. Li, D. Cao, G. Zhang, J. Li, K. Li, Y. Yang, W. Wang, Y. Jin, R. Sun, C.P. Wong, ACS Appl Mater Interfaces 9 (2017) 12147.

[32] A.J. Bandodkar, R. Nunez-Flores, W.Z. Jia, J. Wang, Adv Mater 27 (2015) 3060.

[33] M. Odijk, A.D. van der Meer, D. Levner, H.J. Kim, M.W. van der Helm, L.I. Segerink, J.P. Frimat, G.A. Hamilton, D.E. Ingber, A. van den Berg, Lab Chip 15 (2015) 745.

[34] Y. Xu, X. Xie, Y. Duan, L. Wang, Z. Cheng, J. Cheng, Biosens Bioelectron 77 (2016) 824.

[35] T.B. Tran, S. Cho, J. Min, Biosens Bioelectron 50 (2013) 453. 
[36] Y.C. Xu, Y. Lv, L. Wang, W.L. Xing, J. Cheng, Biosens Bioelectron 32 (2012) 300.

[37] M.W. van der Helm, O.Y.F. Henry, A. Bein, T. Hamkins-Indik, M.J. Cronce, W.D. Leineweber, M. Odijk, A.D. van der Meer, J.C.T. Eijkel, D.E. Ingber, A. van den Berg, L.I. Segerink, Lab Chip 19 (2019) 452.

[38] S.C. Lange, E. van Andel, M.M.J. Smulders, H. Zuilhof, Langmuir 32 (2016) 10199.

[39] A. Weltin, K. Slotwinski, J. Kieninger, I. Moser, G. Jobst, M. Wego, R. Ehret, G.A. Urban, Lab Chip 14 (2014) 138.

[40] S.E. Eklund, R.G. Thompson, R.M. Snider, C.K. Carney, D.W. Wright, J. Wikswo, D.E. Cliffel, Sensors 9 (2009) 2117.

[41] P.M. Misun, J. Rothe, Y.R.F. Schmid, A. Hierlemann, O. Frey, Microsyst Nanoeng 2 (2016) 16022.

[42] V.F. Curto, B. Marchiori, A. Hama, A.M. Pappa, M.P. Ferro, M. Braendlein, J. Rivnay, M. Fiocchi, G.G. Malliaras, M. Ramuz, R.M. Owens, Microsyst Nanoeng 3 (2017) 17028.

[43] D. Bavli, S. Prill, E. Ezra, G. Levy, M. Cohen, M. Vinken, J. Vanfleteren, M. Jaeger, Y. Nahmias, P Natl Acad Sci USA 113 (2016) E2231.

[44] S. Halldorsson, E. Lucumi, R. Gomez-Sjöberg, R.M.T. Fleming, Biosens Bioelectron 63 (2015) 218.

[45] L. Kim, Y.C. Toh, J. Voldman, H. Yu, Lab Chip 7 (2007) 681.

[46] E. Berthier, E.W.K. Young, D. Beebe, Lab Chip 12 (2012) 1224

[47] T. Schulze, K. Mattern, E. Früh, L. Hecht, I. Rustenbeck, A. Dietzel, Biomed Microdevices 19 (2017)

[48] K. Loutherback, L. Chen, H.Y.N. Holman, Anal Chem 87 (2015) 4601.

[49] P. Shah, J.V. Fritz, E. Glaab, M.S. Desai, K. Greenhalgh, A. Frachet, M. Niegowska, M. Estes, C. Jäger, C. Seguin-Devaux, F. Zenhausern, P. Wilmes, Nat Commun 7 (2016) 11535.

[50] S.M. Bonk, M. Stubbe, S.M. Buehler, C. Tautorat, W. Baumann, E.D. Klinkenberg, J. Gimsa, Biosensors-Basel 5 (2015) 513.

[51] P.E. Oomen, M.D. Skolimowski, E. Verpoorte, Lab Chip 16 (2016) 3394

[52] Y.S. Zhang, J. Aleman, S.R. Shin, T. Kilic, D. Kim, S.A.M. Shaegh, S. Massa, R. Riahi, S. Chae, N. Hu, H. Avci, W. Zhang, A. Silvestri, A.S. Nezhad, A. Manbohi, F. De Ferrari, A. Polini, G. Calzone, N. Shaikh, P. Alerasool, E. Budina, J. Kang, N. Bhise, J. Ribas, A. Pourmand, A. Skardal, T. Shupe, C.E. Bishop, M.R. Dokmeci, A. Atala, A. Khademhosseini, P Natl Acad Sci USA 114 (2017) E2293.

[53] F. Hafner, Biosens Bioelectron 15 (2000) 149.

[54] N. Hu, C.X. Wu, D. Ha, T.X. Wang, Q.J. Liu, P. Wang, Biosens Bioelectron 40 (2013) 167.

[55] H. Alborzinia, S. Can, P. Holenya, C. Scholl, E. Lederer, I. Kitanovic, S. Wölfl, Plos One 6 (2011)

e19714.

[56] M. Brischwein, E.R. Motrescu, E. Cabala, A.M. Otto, H. Grothe, B. Wolf, Lab Chip 3 (2003) 234.

[57] S.M. Buehler, M. Stubbe, S.M. Bonk, M. Nissen, K. Titipornpun, E.D. Klinkenberg, W. Baumann, J. Gimsa, Micromachines 7 (2016) 106.

[58] M. Lehmann, W. Baumann, M. Brischwein, R. Ehret, M. Kraus, A. Schwinde, M. Bitzenhofer, I. Freund, B. Wolf, Biosens Bioelectron 15 (2000) 117.

[59] N. Hu, D. Ha, C.X. Wu, J. Zhou, D. Kirsanov, A. Legin, P. Wang, Sensor Actuat a-Phys 187 (2012) 50.

[60] S.M. Grist, L. Chrostowski, K.C. Cheung, Sensors 10 (2010) 9286.

[61] A. Sin, K.C. Chin, M.F. Jamil, Y. Kostov, G. Rao, M.L. Shuler, Biotechnol Progr 20 (2004) 338.

[62] C.H. Lee, J. Folz, J.W.Y. Tan, J. Jo, X. Wang, R. Kopelman, Anal Chem 91 (2019) 2561.

[63] C. Ramello, P. Paullier, A. Ould-Dris, M. Monge, C. Legallais, E. Leclerc, Toxicol in Vitro 25 (2011)

1123.

[64] S.R. Shin, Y.S. Zhang, D.J. Kim, A. Manbohi, H. Avci, A. Silvestri, J. Aleman, N. Hu, T. Kilic, W. Keung M. Righi, P. Assawes, H.A. Alhadrami, R.A. Li, M.R. Dokmeci, A. Khademhosseini, Anal Chem 88 (2016) 10019.

[65] X. Lin, Q. Chen, W. Liu, J. Zhang, S. Wang, Z. Lin, J.M. Lin, Sci Rep 5 (2015) 9643.

[66] S.R. Shin, T. Kilic, Y.S. Zhang, H. Avci, N. Hu, D. Kim, C. Branco, J. Aleman, S. Massa, A. Silvestri, J. Kang, A. Desalvo, M.A. Hussaini, S.K. Chae, A. Polini, N. Bhise, M.A. Hussain, H. Lee, M.R. Dokmeci, A. Khademhosseini, Adv Sci 4 (2017) 1600522.

[67] X. Li, M. Soler, C.I. Özdemir, A. Belushkin, F. Yesilköy, H. Altug, Lab Chip 17 (2017) 2208.

[68] J.F. Lee, G.M. Stovall, A.D. Ellington, Curr Opin Chem Biol 10 (2006) 282.

[69] H. Kimura, T. Yamamoto, H. Sakai, Y. Sakai, T. Fujii, Lab Chip 8 (2008) 741.

[70] S. Cho, A. Islas-Robles, A.M. Nicolini, T.J. Monks, J.Y. Yoon, Biosens Bioelectron 86 (2016) 697.

[71] Y.T. Li, S.H. Zhang, X.Y. Wang, X.W. Zhang, A.I. Oleinick, I. Svir, C. Amatore, W.H. Huang, Angew Chem Int Ed Engl 54 (2015) 9313.

[72] M.W. Li, D.M. Spence, R.S. Martin, Electroanal 17 (2005) 1171.

[73] X.J. Feng, B.F. Liu, J.J. Li, X. Liu, Mass Spectrom Rev 34 (2015) 535.

[74] A. Oedit, P. Vulto, R. Ramautar, P.W. Lindenburg, T. Hankemeier, Curr Opin Biotech 31 (2015) 79.

[75] D. Gao, H.X. Liu, Y.Y. Jiang, J.M. Lin, Lab Chip 13 (2013) 3309.

[76] S. Mao, W. Li, Q. Zhang, W. Zhang, Q. Huang, J.M. Lin, Trac-Trend Anal Chem 107 (2018) 43.

[77] D. Gao, H.X. Liu, J.M. Lin, Y.N. Wang, Y.Y. Jiang, Lab Chip 13 (2013) 978.

[78] C.C. Marasco, J.R. Enders, K.T. Seale, J.A. McLean, J.P. Wikswo, Plos One 10 (2015) e0117685.

[79] D. Gao, H.F. Li, N.J. Wang, J.M. Lin, Anal Chem 84 (2012) 9230.

[80] D. Gao, H.B. Wei, G.S. Guo, J.M. Lin, Anal Chem 82 (2010) 5679.

[81] H.B. Wei, H.F. Li, D. Gao, J.M. Lin, Analyst 135 (2010) 2043.

[82] H.B. Wei, H.F. Li, S.F. Mao, J.M. Lin, Anal Chem 83 (2011) 9306.

[83] S.F. Mao, D. Gao, W. Liu, H.B. Wei, J.M. Lin, Lab Chip 12 (2012) 219.

[84] S.F. Mao, J. Zhang, H.F. Li, J.M. Lin, Anal Chem 85 (2013) 868.

[85] J. Zhang, J. Wu, H.F. Li, Q.S. Chen, J.M. Lin, Biosens Bioelectron 68 (2015) 322.

[86] C.E. Dugan, J.P. Grinias, S.D. Parlee, M. El-Azzouny, C.R. Evans, R.T. Kennedy, Anal Bioanal Chem 409 (2017) 169.

[87] Z. Takats, J.M. Wiseman, B. Gologan, R.G. Cooks, Science 306 (2004) 471. 
R.B. Cody, J.A. Laramee, H.D. Durst, Anal Chem 77 (2005) 2297.

J.J. Liu, H. Wang, N.E. Manicke, J.M. Lin, R.G. Cooks, Z. Ouyang, Anal Chem 82 (2010) 2463.

C.W. Klampfl, M. Himmelsbach, Anal Chim Acta 890 (2015) 44.

W. Liu, J.M. Lin, Acs Sensors 1 (2016) 344 (2011) 1197 .

597 [95] Q.S. Chen, Z.Y. He, W. Liu, X.X. Lin, J. Wu, H.F. Li, J.M. Lin, Adv Healthc Mater 4 (2015) 2291.

J. Wu, M. Jie, X. Dong, H. Qi, J.M. Lin, Rapid Commun Mass Spectrom 30 (2016) 80

[97] H. Bouwmeester, P. Brandhoff, H.J.P. Marvin, S. Weigel, R.J.B. Peters, Trends Food Sci Tech 40 (2014)

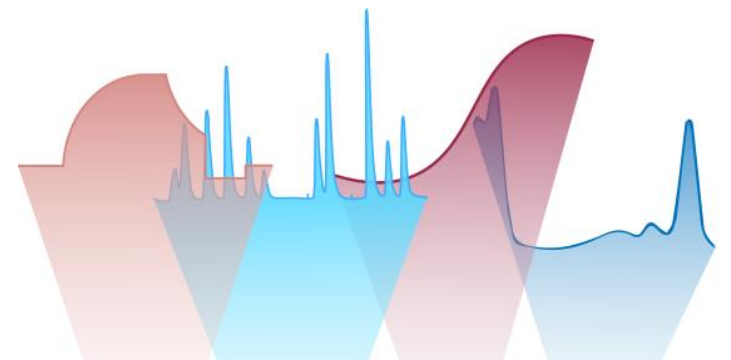

